\title{
Adenovirus en niños con infecciones respiratorias agudas bajas
}

\author{
Dra. Carmen Larrañaga L.. ; QF Manuela Vicente S. 2; Dra. Elba Wu H. ${ }^{\text {; }}$ \\ Dra. Leticia Carrasco S.M,2 ; Dra. Anamar ju Peña D. ${ }^{2}$; Dra. Cecilia Oñate G. ${ }^{2}$; \\ TY Gregorio Aguilera O. ${ }^{2}$ y TM Lilian Vera $D^{2}$

\section{Adenovirus in lower respiratory tract infections}

\begin{abstract}
Seventy eight children were admitted to hospital because of acute lower respiratory tract disease and evidence of infection by adenovirus, in a four year period (1983 through 1986). The presence of adenovirus was detected either by viral jsolation, antigen detection in nasophasyngeal aspirates or serum complement fixation reactions or both. Viral isolations were positive in $66 / 75$ cases. Sixtynine percent of patients were under one year of age. Mixed viral infections ocurred in $43.6 \%$ of all patients and in a significantly higher proportion of those younger than twelve months (51.9\%) as compared with older children $(25 \%)$. Most common symptoms were cough $(93.5 \%)$ fever $(70.5 \%)$ and labored respiration $(70.5 \%)$. More relevant physical signs were tachypnea $(88.4 \%)$, coarse wet rales ( $80.7 \%$ ) wheezing and crepitant rales $(30 \%$ ) and cianosis $(20 \%)$. Mean white blood cell counts were 12,900 (range 2,100-71,000) celis $/ \mathrm{mm}^{3}$ and $1^{\text {st }}$ hour ESR 29 (range 2-78) mm. At Xray examination images suggesting intersticial pneumonia were seen in $84 \%$ of the cases, alveolar densities in $72.4 \%$ and over aeration in $63.7 \%$. Pleural densities and atelectasis were detected in only 10.1 and $7.24 \%$ of these children.
\end{abstract}

(Key words: adenovirus, acute respiratory disease, pneumonia.)

Independientemente de la edad, las infecciones respiratorias agudas obedecen, en su ma. yoría, a agentes virales. De estos, los Adenovirus (Ad.) participan en su etiología en menos de $5 \%{ }^{1,2}$.

La frecuencia de infección respiratoria por Ad. en niños hospitalizados varía en las distintas series entre 4 y $28 \%$; en pacientes pediátricos ambulatorios desciende a $2 \% 1,3$.

La incidencia más alta de enfermedad del aparato respiratorio por este virus se observa en menores de 3 anlos, especialmente entre $\operatorname{los} 4$ y 12 meses (64-75\%), en quienes los cuadros son más graves $\$, 4,5$.

La infección por Ad. produce, a menudo, enfermedad leve del tracto respiratorio alto, pero también puede dar origen a enfermedad broncopulmonar severa, a veces con daño residual o

1. Departamento de Pediatría y Cirugía Infantil, Facultad de Medicina, Dirisión Ciencias Médicas Occidente, Universidad de Chile.

Servicio de Pediatría y Cirugía Infantil, Hospital San Juan de Dios, Area Metropolitana Occidente, Ministerio de Salud.

2. Instituto de Salud Pública de Chile, Sección Virologia. resultado fatal: la letalidad varía entre $12 \mathrm{y}$ $15 \%, 4-11$.

De los 41 serotipos de Ad., los tipos $2,3,4,5$, 7 y 21 con mayor frecuencia se han asociado a infección respiratoria baja y aparecen, además, como productores de complicaciones alejadas. Los hallargos más comunes después de una neumonía severa son bronquiectasias, colapso lobar persistente, fibrosis pulmonar y pulmón hiperlúcido ${ }^{1,7-9,11-13}$.

Entre los factores de riesgo de evolución severa destacan: edad, malnutrición, enfermedades subyacentes, asociación a infección por otros virus y antecedentes de asma previa o de enfermedades respiratorias, especialmente si requirieron de hospitalización ${ }^{4-6,11,12,14-16}$.

Las infecciones respiratorias por Ad. pueden ser de comienzo agudo o, generalmente, insidio$s 0^{4,11,17}$. Los sintomas y signos iniciales son inespecíficos, destacando la fiebre, generalmente más alta y de más larga duración que en otras afecciones virales del aparato respiratorio.

En una o más semanas cobran importancia las manifestaciones respiratorias, que cuando comprometen el tracto respiratorio inferior se presentan frecuentemente como signos bron- 
quiales húmedos $y$ obstructivos $y$, si son severos, semejan crisis asmáticas; aparecen además signos radiológicos de neumonitis, e incluso de condensación, no concordando, muchas veces, los signos clínicos con los hallazgos radiológicos. En ocasiones puede aparecer tos irritativa, persistente y molesta, ocasionando un sindrome co. queluchoideo $4,9,11,18,19$.

Las principales formas clínicas de las infecciones respiratorias agudas bajas por Ad. son bronquitis, bronquiolitis y neumon $\mathrm{ia}^{1,4-6, \mathrm{~B}, 11,12,14}$. El compromiso de otros órganos es frecuente; entre las manifestaciones extrapulmonares se mencionan: encefalomeningitis, miocarditis, pericarditis, hepatitis, daño renal, gastroenteritis, hemorragias, exantemas, coagulación intravascular diseminada, faringitis $y$ conjuntivitis $1,3-7,10,11,15,17,20$.

Debido al poco conocimiento en Chile de la importancia clinica que pueden tener las infecciones por Ad., en el presente trabajo se describen las características personales y clínicas de niños hospitalizados por enfermedad respiratoria aguda baja en los que se detectó este virus y se correlacionan con los resultados virológicos obtenidos.

\section{PACIENTES Y METODOS}

Se estudiaron 78 casos clínicos de infección respiratoria aguda baja en pacientes pediátricos entre 28 días $y$ 13 años y 11 meses de edad, hospitalizados en los años $1983,1984,1985$ y 1986 en el Hospital San Juan de Dios de Santiago de Chile. En todos ellos se había detectado la presencia de Adonovirus: 69,2\% eran menores de un año, la mitad $(51,9 \%)$ tenia entre los 3 y 7 meses de edad (tabla 1) y $58 \%$ cran de sexo masculino.

En todos los niños se realizó un estudio clínico detallado, en una ficha disenada especialmente, que incluyó hemograma, velocidad de eritrosedimentación, gases arteriales y radiografía de tórax.

El estudio virológico se realizó por métodos de aislamiento, detección de antígenos y serología 21,22 . Se consideró que la determinación viral era positiva cuando el virus se detectó por aisłamiento y/o por presencia de ant igenos $y / o$ por serología. Esta última se consideró positiva cuando hubo seroconversión o alza del título de anticuerpos en dos o más diluciones entre el suero agudo y el convalecjente.

El aislamiento en células Hep 2 y fibroblastos de pulmón humano fetal $y$ la presencia de antígeno viral detectado por inmunofluorescencia directa (IF) se hizo a partir de muestras de aspirado nasofatingeo de los pacientes.

El estudio estadístico de las diferencias se hizo con pruebas de $Z$ a un nivel de alfa igual a $5 \%$.

\section{RESULTADOS}

En 34 de los 78 pacientes $(43,6 \%$ ) se identificó además otro virus y en dos se detectó se. roconversión a Mycoplasma pneumoniae. Un caso se asoció a Bordetella pertussis. La frecuencia de las asociaciones virales, según edad, mostró diferencias estad isticamente significativas $(p<0,05)$ entre los menores de un año $(51,9 \%)$ y los mayores de esa edad (25\%) (tabla 1). Los otros virus detectados fueron: Virus respiratorio sincicial $(n=20 ; 58,8 \%)$, miembros de los Pa. rainfluenzae $(n=12 ; 35,3 \%)$, especialmente tipo 3 , virus influenzae $(n=5 ; 14,7 \%)$, herpes simplex $y$ enterovirus en un caso cada uno $(2,9 \%$ respectivamente) y en 7 casos se produjo asociación de Ad. con más de un virus.

El método por el cual se logró identificar el Adenovirus en los 78 casos analizados fue en 75 el aislamiento, en 49 la serología pareada y en 45 la IF directa en frotis de aspirado, ya sea como método único o combinados. De las técnicas utilizadas el aislamiento fue el de mayor rendimiento (66/75 casos positivos), seguido por la serología ( $16 / 49$ positivos) y la IF (14/45 casos) (tabla 2). Trece de los 16 niños con serología positiva eran mayores de 6 meses.

En 28,2\% de los pacientes existía desnutrición en el ingreso; sólo uno ten já desnutrición severa.

Antes del episodio de ingreso al estudio, 57 niños $(73,1 \%)$ habían requerido tratamiento ambulatorio $(n=20)$, hospitalario $(n=25)$ o ambos $(n=12)$. Entre los 37 pacientes que habian sido previamente hospitalizados, 28 $(75,7 \%)$ lo fueron por problemas respiratorios, entre ellos 24 niños sufrieron 50 episodios de

Tabla 1

Distribución de los casos según resultados virológicos por edad $(\%)$

\begin{tabular}{lrrr}
\hline & \multicolumn{2}{c}{ Virus } & \\
\cline { 2 - 3 } & Ad. solo & Mixto* & Total \\
\hline $0-11 \mathrm{ms.} 29$ ds. & $26(48,1)$ & $28(51,9)$ & $54(100)$ \\
$\geqslant 12 \mathrm{~ms}$. & $18(75,0)$ & $6(25,0)^{* *}$ & $24(100)$ \\
\hline Total & $44(56,4)$ & $34(43,6)$ & $78(100)$ \\
\hline
\end{tabular}

* Infección por Ad. con más de un agente(s) viral(es). Fn 2 casos además de otro virus se asoció a M. pneumoniae.

*** $\mathrm{p}<0,05$. 
Tabla 2

Distribución de los casos según técnicas virológicas utilizadas

\begin{tabular}{lrrrr}
\hline \multirow{2}{*}{ Técnica } & & \multicolumn{3}{c}{ Resultados positivos } \\
\cline { 2 - 5 } & & A & S & IFD \\
\hline A + S + IFD & 28 & 23 & 9 & 7 \\
A + S & 19 & 14 & 7 & - \\
A IFD & 16 & 15 & - & 6 \\
A & 12 & 12 & - & - \\
S & 2 & 2 & - & - \\
IFD & 1 & - & - & 1 \\
\hline Total & 78 & 66 & 16 & 14 \\
\hline
\end{tabular}

$A=$ Aislamiento viral.

$\mathrm{S}=$ Serología pareada .

IFD = Inmunofluorescencia directa.

bronconeumonía y 12 tuvieron 26 episodios de obstrucción bronquial, afecciones que con frecuencia ocurrieron en forma concomitante en el mismo enfermo.

Los síntomas clínicos más frecuentes fueron: tos $(93,5 \%$ de los casos), fiebre $(70,5 \%)$ con temperaturas promedio de $38,7^{\circ} \mathrm{C}$ y dificultad respiratoria progresiva $(69,2 \%)$. Se describió "compromiso del estado general" (astenia, anorexia y adinamia) en $43,5 \%$ y cianosis en $24,3 \%$ de los niños.

En el examen físico de ingreso fueron calificados como graves $93,5 \%$, tenían taquipnea $88,4 \%$ (frecuencias respiratorias iguales o mayores a 30 respiraciones por minuto) y cianosis $20 \%$ de los pacientes. Entre los hallazgos pulmonares destacan por su frecuencia los signos bronquiales húmedos $(80,7 \%)$ y la dificultad respiratoria, caracterizada por retracción torácica $(69,2 \%)$. En 30\% de los pacientes se encontraron signos de obstrucción y condensación pulmonar. Las manifestaciones extrapulmonares más relevantes fueron higado palpable, faringitis $\mathrm{y}$ adcnopatias, de predominio cervical.

En cuatro enfermos existieron hechos poco frecuentes, pero significativos: uno tenía signos meníngeos, otro miopatía, el tercero ictericia y el último síndrome de Reye confirmado.

El hemograma de los 78 casos mostró leve tendencia a la anemia con hematocrito promedio de $33,4 \%$. El recuento promedio de blancos fue de $12.900 / \mathrm{mm}^{3}$ (max.: 71.000 min.: 2.100). No hubo diferencias significativas entre los polimorfonucleares y mononucleares.
En la mitad de los casos ocurrió desviación a izquierda leve a moderada. La velocidad de eritrosedimentación presentó aumentos discretos: promedio $29 \mathrm{~mm}$, rango 2 a $78 \mathrm{~mm}$ en la primera hora. Los gases arteriales cursaron en general con hipoxemias leves a moderadas. En las radiografias de tórax efectuadas en 69 pacientes se registraron imágenes intersticiales sugerentes de neumonitis en $58(84 \%)$ niños, signos de condensación en 50 $(72,4 \%)$; hiperinsuflación pulmonar en 44 $(63,7 \%)$; las imágenes de reacción pleural y atelectasias fueron mucho menos frecuentes, detectándose, respectivamente, en $7(10,1 \%)$ y 5 $(7,24 \%)$ de los casos.

Bronconeumonía fue el diagnóstico de egreso más frecuente, seguido de bronquitis obstructiva y neumonitis. En el $9 \%$ de los ninos con infecciones por Ad. se diagnosticó sindrome coqueluchoideo, y en uno de estos casos se demostró infección por Bordetella pertussis (tabla 3 ).

Tabla 3

Diagnósticos respiratorios de egreso en 78 pacientes con adenoyirus

\begin{tabular}{lrr}
\hline & \multicolumn{2}{c}{ Egreso } \\
\cline { 2 - 3 } Diagnóstico* & $n$ & \\
\hline Bronconeumonía & 49 & 62,8 \\
Enf. bronquial obstructiva aguda & 40 & 51,3 \\
Neumonitis & 33 & 42,3 \\
Síndrome coqueluchoideo & 7 & 9,0 \\
Atelectasia & 6 & 7,7 \\
Pleuroneumonía & 4 & 5,1 \\
Neumopatía postexantemática & 2 & 2,6 \\
Neumopat ía crónica & 4 & 5,1
\end{tabular}

* 53 pacientes presentaron asociación de patologja respiratoria. La asociación más frecuente fue bronconeumon ía y bronquitis obstructiva.

Cinco pacientes presentaron manifestaciones de compromiso encefálico en su evolución, 2 fallecieron.

De los 78 niños, 6 fallecieron, 5 por causas relacionadas con la infección por Ad.

\section{DISCUSION}

En el presente trabajo se relacionan las características personales, clínicas y virológicas en pacientes pediátricos hospitalizados por infección respiratoria aguda en quienes se detectó Ad. 
La mayoría de éstos eran menores de un año, agrupándose especialmente entre 3 y 7 meses de edad, lo que concuerda con otros estudios ${ }^{1,4,5}$. L.lama la atención el alto porcentaje de casos con infecciones virales mixtas, cuya prevalencia es estadisticamente significativa en menores de un affo. Las asociaciones de $\mathrm{Ad}$. con otros virus. especialmente con VRS y Parainfluenza, pero también con otros agentes no virales, ha sido señalado como posibles agravantes de la severidad de la enfermedad ${ }^{11,12,14,23} ;$ lo que en nuestro trabajo no se confirmó. Sin embargo, los dos enfermos que cursaron ademas con infección por Mycoplasma pneumoniae fueron casos graves; lo mismo ocurrió con otros dos niños que evolucionaron simultáneamente con varicela. EI hecho de no contar con estudios bacteriológicos simultáneos de todos los enfermos impide aclarar si los casos de mayor gravedad pudieran relacionarse con sobreinfecciones bacterianas.

De los 78 pacientes, seis fallecieron y en cinco el deceso se relacionó directamente con la infección respiratoria. En dos, la causa aparente fue sólo la infección por Ad., ya que no se detectó ningún otro agente patógeno; en un caso se produjo infección viral mixta sin complicaciones bacterianas y los dos nifros restantes se sobreinfectaron con gérmenes de origen intrahtospitalario, pesquisados después de identificar el adenovirus.

Es importante destacar que los cuadros graves, en que además se detectaron agentes no virales, no respondieron a múltiples esquemas de antibióticos, aunque fueran sensibles in vitro. Este hecho concuerda con apreciaciones que sentalan que los $A d$., solos o asuciados con otros virus, tienen la suficiente capacidad para producir enfermedades severas $s^{4,6,12,14,24}$.

La desnutrición en nuestro estudio no pudo considerarse como factor de riesgo, ya que sólo en un niño fue avanzadz: en el resto de los casos en que se encontró era leve y no guardó relación con la gravedad de la enfermedad.

El antecedente de afecciones respiratorias es un factor de riesgo importante en la adquisición de infección respiratoria aguda grave por Adenovirus ${ }^{11,14}$. Aunque este antecedente puede estar influido por la alta incidencia, en general, de infecciones respiratorias agudas en los niños, especialmente en los menores de un año, debemos señalar que todos los fallecidos habían tenido episodios reiterados de obstrucción bronquial.
Si bien la mayoría de los síntomas y signos observados ocurren tambjén en otros cuadros respiratorios, su modo de presentación y evolución se atiene a los descrito en las infecciones por Ad.: comienzo insidioso, promedio 6,6 días de evolución antes del ingreso y hospitalización prolongada, promedio 18,5 días debido a la gravedad de muchos de los pacientes.

La mayor frecuencia de bronconeumonia como forma clínica predominante está de acuerdo con otras publicaciones ${ }^{1,6,8,11}$. La neumonitis se diagnosticó con menor frecuencia que lo esperado, probablemente por omisión, ya que el porcentaje de imágenes compatibles encontradas en el estudio radiológico fue alto.

Las variaciones importantes en el recuento de leucocitos fueron señaladas en otros traba$\operatorname{jos}^{4}, 9,11,17$. La tendencia a leucopenia en las formas más graves, la aparición de linfocitos hiperbasófilos y las formas juveniles en cifras bajas a moderadas orientan más hacia la etiología viral. Este examen resulta más orientador cuando se le interpreta junto con las manifestaciones clínicas y radiológicas: el diagnóstico diferencial más frecuente y dificil de la neumonía por Ad. son las neumonías bacterianas.

Las imágenes radiológicas encontradas, especialmente las de condensación, confirman que ellas no son exclusivas de las infecciones bacterianas. La hiperinsuflación pulmonar, presente en más de la mitad de los enfermos, es uno de los signos que aparece en el pulmón hiperlúcido, entidad radiológica y clínica descrita como secuela de infección respiratoria de etiología viral, fundamentalmente por Ad, ${ }^{: 5,11}$.

Los casos analizados se detectaron en los años 1983 a 1986. Los Ad. estuvieron presentes en todos los meses estudiados, pero su predominio en el mes de agosto correspondió a un mayor número de casos en dicho mes durante 1984 y 1986, años en que hubo en Santiago brotes epidémicos de infecciones por estos virus, y mayor frecuencia de cuadros respiratorios graves que requirieron de hospitalización.

La técnica de mayor rendimiento en la detección de Ad. fue el aislamiento viral a partir de aspirados nasofaringeos, que es la muestra ideal para el aislamiento y la detección de antígenos virales ${ }^{23}, 24$. A pesar de que otros investigadores consideran las deposiciones más útiles, a nuestro juicio esto es errado, pues se ha demostrado que varios tipos de Ad. pueden excretarse durante 
mucho tiempo por esta via y no indicar necesariamente infección reciente ${ }^{1,24}$.

La serología no tuvo buen rendimiento; especialmente se observaron bajos indices de seroconversión en los menores de un año, como ha ocurrido en otras investigaciones ${ }^{23,24}$.

Aunque no obtuvimos gran positividad con IF, esta es una técnica muy específica para detección de antígenos directamente en células del aparato respiratorio, y de rápida ejecución, siendo la más conveniente para aplicar en el manejo clínico de pacientes. Es muy importante que la obtención de las muestras para IF y aislamiento se haga temprano en la evolución de la enfermedad ${ }^{21,24}$.

Los Ad. son especialmente aptos para producir infecciones nosocomiales; de allí la importancia de su identificación rápida en los casos hospitalizados, para minimizar el riesgo de expansión.

Por la edad en que se detectó la mayoria de los Ad., las características clinicas y radiológicas de los cuadros respiratorios producidos, creemos que los Ad. identificados representan, en un gran porcentaje, primoinfecciones con posible importancia etiológica y no reactivaciones de $\mathrm{Ad}$. latentes. Como no se realizó un estudio acabado de la etiología, no podemos descartar la participación bacteriana o de otros agentes simultáneamente en alguna proporción de nuestros pa. cientes.

Los Ad, detectados en este estudio no fueron tipificados, to que consideramos de gran importancia para estudios futuros, pues ello nos ofrecería un panorama más exacto de la presencia de estos virus y también de los tipos ge. nómicos involucrados en las patologías más severas $^{1}$.

\section{RESUMEN}

Se estudiaron 78 casos clínicos hospitalizados por infección respiratoria aguda baja durante los aflos 1983 a 1986. En todos se confirmó Adenovirus (Ad.) por aislamiento viral, detección de antígeno en aspirado nasofaringeo o serología pareada.

De las técnicas para diagnóstico virológico empleadas, el aislamiento viral tue la de mayor positividad (66/78 casos).

Se analizaron las características personales de los pacientes, factores de riesgo de adquirir in- fección grave por Ad., manifestaciones clínicas y resultados virológicos obtenidos.

De los 78 casos estudiados, el $69,2 \%$ eran menores de un año. En el 43,6\% de los enfermos ocurrió infección virl mixta, la que prevaleció significativamente tambièn en menores de un año.

Uno de los factores de riesgo presente con mayor frecuencia fue el antecedente de patología respiratoria, ya sea ambulatoria, hospitalaria o ambas.

La evolución clínica fue generalmente larga y con las características ya conocidas para las infecciones por Ad.

Entre los exámenes de laboratorio destacan: lo poco característico del hemograma, la tendencia a la hipoxemia y las alteraciones radiológicas con una alta frecuencia de imágenes de neumonitis, condensación e hiperinsuflación pulmonar.

La letalidad de los casos estudiados fue de $7,7 \%$ (6 casos).

\section{REFERENCIAS}

1. Wadell G.: Adenoviruses. Principles and Practice of Clinical Virology. Edit.: Zuckerman A.J., Benatvala J.E., Pattison J.R., John Wiley \& Sons Ltd., London. 1987.

2. Jackson E.E., Huldoon R.L.: Viruses causing common Iespiratory infection in man. IV. Reoviruses and Adenoviruses. J Infect Dis 1973; 128: 811.66 .

3. V'an der Veen $J$ : The role of Adenoviruses in respiratory disease. Am Rev Respir 1963;88: 167-80.

4. Chin-Hsien $T$ : Adenovirus pneumonia epidemic among Peking infants and preschool chitdren in 1958. Chin Med J (Engl) 1960; 80: 331-9.

5. Wenman W.M., Pagtakhan R.D., Reed M.H., Chemick V.. Albritton W.: Adenovius Bronchiolitis in Manitoba: Epidemiologic, Clinical and Radiologic Features. Chest 1982; May B1(5): 605-9.

6. Chany C. Lepine R., Vinh Lt., Satge P., Virat $J$.: Severe and fatal pneumonia in infants and young children associated with Adenovirus infection. Am J Hyg 1958: 67 : 367-78.

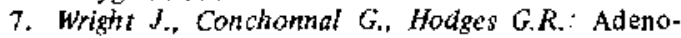
virus type 21 infection. Occurrence with pneumonia, rhabdomyolisis and myoglobinuria in an aduli. J A M A 1979;241: 2420-21.

8. Becroft DMO. Bronchiolitis obliterans bronchiectasis and other sequelas of Adenovirus type 21 infection in young children. I Clin Pathol 1971; 24: 72 .

9. Leers W.D., Sarin M.K., Kasupski G.Y.: Lobur Pneumonia associated with Adenovirus type 7. Can Med A ssoc J $1981 ; 125(9)$ : 1003-4. 
10. Levy $Y_{.}$, Nitzan $H_{+}$Beharab $A$. et al.: Adenovirus type 3 infection with systemic manifestation in apparently notmal children. lsc J Med Sci 1986; 22(11): 774-8.

11. Spiegelbiatt L., Rosenfeld R.: Hiperlucent lung: long-term complication of Adenovirus type 7 pneumonia. Can Med Assoc J 1983; $128: 47-9$.

12. Herbert F.A., Wilkinson D., Burchak E., Morgante $O$.: Adenovirus type 3 pneumonia causing lung damage in chilhood. Can Med Assoc J 1977; 116: 274-6.

13. Letin S., Dietrich J., Guillory J.: Fatal nonbacterial pneumonia associated with Adenovirus type 4. Occurence in an adults. J A M A 1967; 201: 975-7.

14. Straube R.C.; Thompson M.A., Von Dyke R.B. et al.: Adenovirus type 7 b in a Children's Hospital. J Infect Dis $1983 ; 147(5)$ : 814-9.

15. Harris D.J., Wulff $H_{.}$, Ray C.E., Poland J.D., Chin T.D.Y. Wenner H.A.: Viruses and diseases III. An outbreak of Adenovirus type $7 \mathrm{a}$ in a children's home. Am J Epidemiol 1971;93: 399-402.

16. Freiman 1, Super M., Joosting A.C.C., Harwin R.M. Gear I.H.S.: An Lipidemic of Adenovirus type 7 bronchopneumonia in Bantu children. S Afr Med I 1971;45:107-11.
17. $\operatorname{Kim}$ K.S., Godh R.S.: Acute Encephalopathy in Twins due to Adenovirus type 7 infection. Arch Neurol $1983 ; 40(1)$ : $58-9$.

18. H'u $E$, Avendaño F., Grado $C$ : Síndrome Coqueluchoideo y Adenovirus. I. Rev Chil Pediatr 1975; 46: $34-46$.

19. Avendaño L.F., Wu E., Grado C.: Síndrome Coqueluchoideo y Adenovirus. II. Estudjo de Laboratorio. Rev Chil Pediatr 1975;46:307.

20. Richmond S.J., Caul E.O., Dunn S.M., Ashley C.R., Charke S.K.R., Seymour N.R.: An outbreak of gastroen1eritis in young children caused by Adenoviruses. Lancet 1979; 1: 1178-80.

21. Gardner P.S. McQuillan $J_{\text {.; }}$ Application of immunofluorescent antibody technique in the rapid diagnosis of respiratory syncityal virus infection. Br Med J 1968; 3 : 340-3.

22. Siver J.L.: Application of a microtechnique to viral serological investigations. J Immunol 1962; 88: 320.9 .

23. Paisley I.W., Lauer B.A., McIntosh K. et al.: Pathogens associated with acute lower respiratory tract infection in young children. Pediztr lnfect Dis $1984 ; 3(1): 14-9$.

24. Ruuskanen $O$, Metuerman O., Sarkkinen $H$.: Adenoviral Diseases in Children: A study of $105 \mathrm{Hos}$ pital cases. Pediatrics 1985; 76(1): 79-82. 